\title{
Double-Blind Randomized Trial on the Efficacy of the Forced Prolonged Position for Treatment of Lateral Canal Benign Paroxysmal Positional Vertigo
}

\author{
Marco Mandalà, MD, PhD; Luigi Califano, MD; Augusto Pietro Casani, MD; Mario Faralli, MD; \\ Vincenzo Marcelli, MD; Giampiero Neri, MD; Rudi Pecci, MD; Felice Scasso, MD; \\ Leonardo Scotto di Santillo, MD; Paolo Vannucchi, MD; Beatrice Giannoni, MD; \\ Soumit Dasgupta, MBBS MSc FRCS FRCP; Ilaria Bindi, MD (1); Lorenzo Salerni, MD, PhD; \\ Daniele Nuti, MD
}

\begin{abstract}
Objectives/Hypothesis: The need for class I and II studies on the efficacy of liberatory maneuvers in the treatment of lateral canal benign paroxysmal positional vertigo (LC-BPPV) motivated the present double-blind randomized trial on the short-term efficacy of the forced prolonged position (FPP).

Study Design: Double-blind, randomized controlled trial.

Methods: Two hundred twenty-one patients with unilateral LC-BPPV met the inclusion criteria for a multicentric study. Patients were randomly assigned to treatment by FPP (116 subjects) or sham treatment (105 subjects). Subjects were followed up at 24 hours with the supine roll test by blinded examiners.

Results: Among the sample, $67.4 \%$ and $32.6 \%$ of the patients showed respectively geotropic and apogeotropic variant of LC-BPPV. At the 24-hour follow-up, the effectiveness of FFP compared to the sham maneuver was, respectively, $57.8 \%$ versus $12.4 \%(P<.0001)$ in the total sample, $76.9 \%$ versus $11.3 \%(P<.0001)$ in the geotropic variant group, and $60.5 \%$ versus $17.6 \%(P=.0003)$ in the apogeotropic variant group, including resolution or transformation to geotropic variant.

Conclusions: FPP proved highly effective compared to the sham maneuver. The present class 2 study of the efficacy of the FPP changes the level of recommendation of the method for treating LC-BPPV into a strong one.

Key Words: Benign paroxysmal positional vertigo, forced prolonged position maneuver, evidence based, double-blind randomized trial, vestibular, semicircular canals, lateral canal.
\end{abstract}

Level of Evidence: 2

Laryngoscope, 00:1-5, 2020

\section{INTRODUCTION}

Benign paroxysmal positional vertigo (BPPV) is a well-known labyrinthine disorder caused by a mechanic stimulation of the vestibular receptors. It is characterized by positional vertigo and positional nystagmus (PN), both provoked by changes in the position of the head with

From the Department of Otolaryngology (M.M., I.B., L.S., D.N.), Azienda Ospedaliera Universitaria Senese, Siena, Italy; Audiology and Phoniatrics Unit (L.C.), Ospedale G. Rummo, Benevento, Italy; Department of Neuroscience, Otorhinolaryngology Unit (A.P.C.), University of Pisa, Pisa, Italy; ENT Department (M.F.), University of Perugia, Perugia, Italy; San Giovanni Bosco Hospital (v.M.), Naples, Italy; Department of Neuroscience, Imaging and Clinical Sciences, Otorhinolaryngology Unit (G.N.), University of Chieti, Chieti, Italy; Department of Oto-Neuro-Ophthalmology, Audiology Unit (R.P., P.V., B.G.), University of Florence, Florence, Italy; U.O. ORL, Ospedale P A MiconeASL 3 (F.S., L.S.D.S.), Genova, Italy; Department of Audiovestibular Medicine and Neurotology (S.D.), Alder Hey Children's Hospital NHS

Trust, Liverpool, United Kingdom; and the Sheffield Vertigo and Balance Centre (S.D.), Sheffield, United Kingdom.

8,2020 .

Editor's Note: This Manuscript was accepted for publication on July

The authors have no funding, financial relationships, or conflicts of interest to disclose.

Send correspondence to Marco Mandalà, $\mathrm{MD}, \mathrm{PhD}$, V. le Bracci, 1653100 Siena, Italy. E-mail: marcomand@hotmail.com

DOI: 10.1002/lary.28981 respect to gravity. The lifetime prevalence of BPPV is recently estimated at $2.4 \%$, the 1 -year prevalence is $1.6 \%$, and the 4 -week prevalence is $0.7 \%$. Posterior semicircular canal BPPV (PC-BPPV) is the most common form, whereas lateral semicircular canal BPPV (LC-BPPV) is the second-most common type of BPPV in about $15 \%$ to $20 \%$ of patients. $^{1-3}$

LC-BPPV is characterized by horizontal directionchanging PN induced by head turning while supine. Depending on the different locations of otoconial debris in the canal, LC-BPPV can be divided into two variants, the more common with geotropic nystagmus and the less common one with apogeotropic nystagmus. With both of the two variants, the pathological side is identified by the more intense PN. The supine roll test is performed by rolling the patient's head $90^{\circ}$ to one side to trigger the PN. Geotropic nystagmus is more intense when the pathological ear is lowermost; the apogeotropic variant is characterized by more intense PN when the affected ear is uppermost. Horizontal positional nystagmus has a short latency and prolonged duration with poor fatigability. ${ }^{4-7}$ This test is the best method of diagnosing LC-BPPV. 
Treatment of BPPV relies on physical maneuvers and positions that are immediately effective in many patients. The aim of physical therapy is to dislodge the otoconial debris from the semicircular canals by gravitation and/or centrifugal inertia. Many maneuvers have been devised for the treatment of LC-BPPV, beginning with the barbecue rotation by Lempert. ${ }^{8}$ The liberatory maneuver devised by Gufoni (GLM) is a kind of technique whose efficacy has been validated with at least three class 1 studies (randomized double-blind study). ${ }^{9-11}$ The GLM requires that the patient is quickly brought from the sitting to the lateral position, on one shoulder, to allow the particles to exit the canal by the centrifugal force created by the rapid deceleration. GLM is a good and effective option for most patients and has the great advantage, when successful, to cure the patients instantaneously by moving the particles. However, there are some circumstances where it is difficult to perform, for example in older people, obese patients, or in those with significant mobility limitations. Furthermore, there are some LCBPPV patients where the diagnostic maneuvers provoke severe autonomic symptoms that preclude completing the head movement. One of the effective options for these patients may be the so called forced prolonged position (FPP), devised by Vannucchi et al. ${ }^{12}$

The FPP is a very simple and easy method that should allow debris to exit the canal under gravitation. Once the pathologic side has been identified by the direction of most intense PN, the patient is simply instructed to lie in a supine position, then to turn on to the side of the healthy ear (geotropic variant) or pathological side (apogeotropic variant) and to stay in that position for as long as possible preferably all night. This facilitates otoconial debris exiting the canal under gravity. Using this approach, a remission rate ranging from $75 \%$ to $90 \%$ is obtained. ${ }^{13-15}$ The results obtained by FPP are based on class IV studies, and the strength of recommendation is not strong.

The need to determine the evidence-based efficacy of FPP in LC-BPPV, as recommended by the BPPV guidelines produced by the American Academies of Neurology and Otolaryngology and Barany Society, ${ }^{16,17}$ motivated the present multicentric, double-blind, randomized controlled trial. The aim was also to improve the evidencebased efficacy of LC-BPPV maneuvers to develop strong recommendations for clinical practice and formulate guidelines.

\section{MATERIALS AND METHODS}

Two hundred forty-six subjects were diagnosed with unilateral LC-BPPV based on typical PN in a multicentric study that involved eight vestibular units in Italy (Benevento, Chieti, Firenze, Genova, Napoli, Perugia, Pisa, and Siena) from June 2016 to June 2017. The following inclusion criteria for recruitment into this study was determined by the Barany Society Criteria for LC-BPPV ${ }^{6,18}$ :

1. Recurrent attacks of positional vertigo or positional dizziness provoked by lying down or turning over in the supine position

2. Duration of attacks $<1$ minute for geotropic form and $>1$ minute for apogeotropic form
3. Positional nystagmus elicited after a brief latency or no latency by the supine roll test, beating horizontally toward the undermost/uppermost ear with the head turned to either side (geotropic/apogeotropic direction changing nystagmus), and lasting $<1$ minute (geotropic variant) and $>1$ minute (apogeotropic variant).

4. Not attributable to another disorder.

An additional inclusion criterion was the visual evident differences in nystagmus intensity between two sides, allowing the identification of the affected side.

Exclusion criteria were diagnosis of multiple canals or atypical PN, diagnosis of other peripheral vestibular disorders (e.g., vestibular neuritis, Menière's disease, enlarged vestibular aqueduct syndrome, and third window disorders), vestibular migraine, and central vestibular system diseases.

Based on the exclusion criteria, out of 246 subjects, seven patients were lost at follow-up. Two patients $(0.81 \%)$ with the apogeotropic variant received a diagnosis of central vestibular disorders, three subjects $(1.21 \%)$ presented with multiple canal involvement, eight patients $(3.25 \%)$ demonstrated peripheral vestibular disorder comorbidities, and in five subjects $(2.03 \%)$ a clear preponderance of the positional nystagmus toward one side with the supine head roll test was found. These patients were excluded from the study. Therefore, based on the inclusion and the exclusion criteria, a total of 221 subjects were enrolled in the present study. All 221 patients met the inclusion criteria. The sample size (number of patients enrolled) per site was: Benevento 41, Chieti 24, Firenze 23, Genova 21, Napoli 27, Perugia 27, Pisa 26, and Siena 32.

All patients received neurotologic examination, including videonystagmography recordings of spontaneous and gazeevoked nystagmus, vertical and horizontal smooth pursuit, and saccades, limb ataxia, and balance function. Informed consent was obtained from all participants. Diagnosis was performed with evidence of PN induced by bringing the patient first from sitting to supine position and then by turning their heads $90^{\circ}$ to either side while in the supine position (supine head roll test). To exclude BPPV of the anterior/posterior canal, ${ }^{6}$ both the Dix-Hallpike test and supine straight head-hanging position ${ }^{6}$ (bringing the patient to the supine position with head extended by $30^{\circ}$ or more) were also performed. The affected ear was identified by the direction of the more intense PN, that is the undermost ear with geotropic and the uppermost ear with apogeotropic PN. The positional nystagmus was not quantified by videonystagmography.

A second blinded investigator obtained a randomly computer-generated eight-block code to treatment with FPP (116 subjects) or sham treatment (105 subjects). In the geotropic group, the correct procedure was to lie with the affected ear up and the sham procedure with the affected ear down, whereas in the apogeotropic group, the correct procedure was with the affected ear down and sham procedure with the affected ear up.

The expected responses to $\mathrm{PN}$ following prolonged bed rest on one side were:

1. Geotropic group: recovery (absence of vertigo and nystagmus, treatment group) if FPP with the affected ear up or no variation or conversion to apogeotropic PN if affected ear down (sham group)

2. Apogeotropic form: conversion to geotropic PN or recovery with the affected ear down (treatment group) or no variation with the affected ear up (sham group).

The expected responses to a prolonged bedrest on one side (FPP) were:

1. Geotropic group: recovery (absence of vertigo and nystagmus) with the correct position (affected ear up) and no variation or 
conversion to the apogeotropic variant with the sham position (affected ear down)

2. Apogeotropic group: conversion to the geotropic variant or recovery with the correct position (affected ear down) and no variation with the sham position (affected ear up)

The second blinded investigator then advised the patients to go home and verbally instructed the side on which to adopt the FPP; that is, at the time of going to bed for the night they had to lie down with their head straight, then to turn on one side and possibly to stay on that position for all the night. In the event of awakening or getting out of bed, they had to reassume the same position.

The treatment outcome was re-evaluated the following day (24-hour follow-up) by a third blinded investigator in each unit, who repeated the supine head roll test, and if no PN was found, the Dix-Hallpike maneuver was performed. Patients without PN were considered as recovered and asked to return in case of recurrence of positional vertigo in the first week after treatment. Patients who still demonstrated LC-BPPV or PC-BPPV were treated according to the pattern of positional nystagmus.

The study was approved by the local ethics committees of all hospitals where the clinical trial was conducted. The registration number is 3/09 (year 2009) in the registry of Comitato Etico Locale, Via delle Scotte, 14-53100 Siena, approved in Siena, Italy, February 24, 2009. The full trial protocol can be accessed and requested at the ENT Department, University of Siena, Viale Bracci, 11, 53100, Siena, Italy, or from the corresponding author (M.M.).

Calculation of the sample size can be found in a previous article and was based on the assumption of a minimum difference of $35 \%$ in the primary outcome between the groups (FPP success rate $85 \%$ vs. sham treatment success rate $50 \%$ ), with an $\alpha$ of $5 \%$, and a discriminating power of $80 \%$. The minimum number of patients required in each treatment arm was 27. Comparisons between groups were assessed by the Fisher exact test or $t$ test, as appropriate, at a significance level of $P<.01$. Statistical analysis was performed with SPSS software (IBM, Armonk, NY).

\section{RESULTS}

Demographic and clinical data of the population studied are presented in Table I. The mean age, sex preponderance, and side affected of the whole population studied (221 patients) were, respectively, $58.84 \pm 16.94$ years, 81/140 (male/female, 0.58 ratio), and 126/95 (right/left side).

Among the 221 patients of the study population, $149(67.4 \%)$ showed an LC-BPPV geotropic variant, and $72(32.6 \%)$ an apogeotropic form. No significant differences were found in terms of age and sex preponderance

\begin{tabular}{|c|c|c|c|}
\hline \multicolumn{4}{|c|}{$\begin{array}{l}\text { TABLE I. } \\
\text { Demographic and Clinical Data of the Study Population. }\end{array}$} \\
\hline & $\begin{array}{l}\text { Forced Prolonged Liberatory } \\
\text { Maneuver, } 116 \text { Subjects }\end{array}$ & $\begin{array}{l}\text { Sham Maneuver, } \\
105 \text { Subjects }\end{array}$ & $P$ Value \\
\hline Age, yr & $58.32 \pm 15.62[15-81]$ & $60.48 \pm 17.13[42-82]$ & .5328 \\
\hline Sex, M/F & $44 / 72$ & $37 / 68$ & .6743 \\
\hline Side, R/L & $67 / 49$ & $59 / 46$ & .6915 \\
\hline Type, geotropic/apogeotropic & $78 / 38$ & $71 / 34$ & 1.0000 \\
\hline Onset of BPPV, $d$ & $6.8 \pm 8.1$ & $8.1 \pm 10.9$ & .1635 \\
\hline Previous episodes of BPPV, Y/N & $35 / 81$ & $28 / 77$ & .6547 \\
\hline Total, 221 subjects & $67 / 49$ & $13 / 92$ & $<.0001$ \\
\hline \multicolumn{4}{|c|}{$\begin{array}{l}\text { 24-hour control (absence/presence of positional } \\
\text { nystagmus) }\end{array}$} \\
\hline \multicolumn{4}{|l|}{ Nystagmus presence } \\
\hline Unchanged & 28 & 81 & \\
\hline LC-BPPV transformation & 17 & 6 & \\
\hline PC-BPPV transformation & 4 & 5 & \\
\hline Geotropic variant, 149 subjects & $60 / 18$ & $8 / 63$ & $<.0001$ \\
\hline \multicolumn{4}{|c|}{$\begin{array}{l}\text { 24-hour control (absence/presence of positional } \\
\text { nystagmus) }\end{array}$} \\
\hline \multicolumn{4}{|l|}{ Nystagmus presence } \\
\hline Unchanged & 16 & 54 & \\
\hline APOGEO LC-BPPV transformation & 1 & 5 & \\
\hline PC-BPPV & 1 & 4 & \\
\hline Apogeotropic variant, 72 subjects & $7 / 31$ & $5 / 29$ & .7583 \\
\hline \multicolumn{4}{|c|}{$\begin{array}{l}\text { 24-hour control (absence/presence of positional } \\
\text { nystagmus [nystagmus transformation]) }\end{array}$} \\
\hline \multicolumn{4}{|l|}{ Nystagmus presence } \\
\hline Unchanged & 12 & 27 & \\
\hline GEO LC-BPPV transformation & 16 & 1 & \\
\hline PC-BPPV & 3 & 1 & \\
\hline
\end{tabular}

APOGEO LC-BPPV = apogeotropic lateral canal benign paroxysmal positional vertigo; BPPV = benign paroxysmal positional vertigo; GEO LC-BPPV = geotropic lateral canal benign paroxysmal positional vertigo; LC-BPPV = lateral canal benign paroxysmal positional vertigo; $\mathrm{M} / \mathrm{F}=\mathrm{male} / \mathrm{female}$; $\mathrm{PC}-\mathrm{BPPV}=\mathrm{posterior}$ canal benign paroxysmal positional vertigo; $\mathrm{R} / \mathrm{L}=\mathrm{right} / \mathrm{left} ; \mathrm{Y} / \mathrm{N}=\mathrm{yes} / \mathrm{no}$. 


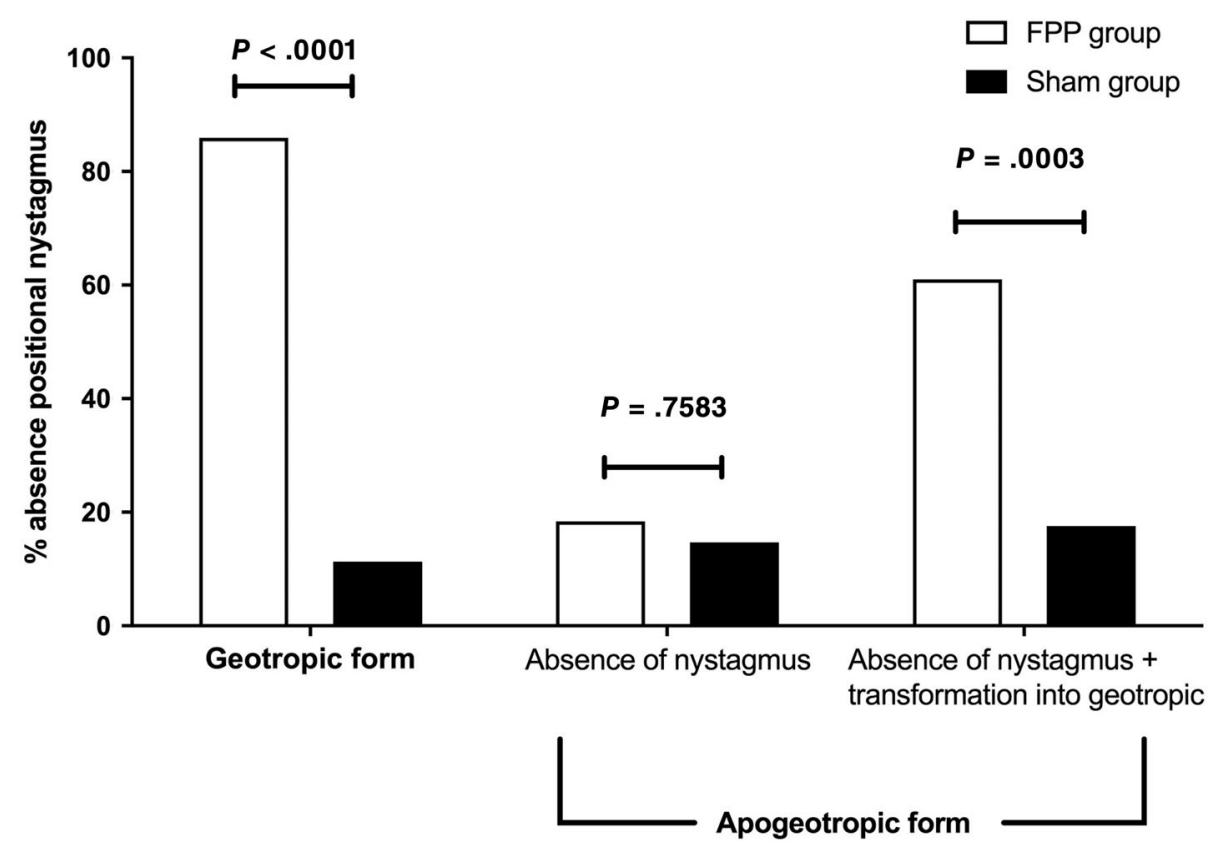

Fig. 1. Twenty-four-hour follow-up efficacy of the forced prolonged position (FPP) versus sham maneuver in patients with geotropic and apogeotropic lateral canal benign paroxysmal positional vertigo. In the apogeotropic form, results are presented according to absence of positional nystagmus $(\mathrm{PN})$ and absence of $\mathrm{PN}$ plus transformation to geotropic variant.

among the treatment and sham groups $(P>.05)$. The two randomized groups were also comparable regarding previous episodes of BPPV, side affected, and mean duration of symptoms before the first visit $(3.16 \pm 3.76$ days, $P>$.05). Most patients were examined within 5 days from the symptom's onset (174/221, 78.7\%).

At the 24-hour follow-up, 67/116 (57.8\%) of patients undergoing FPP (both geotropic and apogeotropic variant) had recovered from positional nystagmus and vertigo, compared to $13 / 105(12.4 \%)$ of patients undergoing the sham maneuver $(P<.0001)$. No patients reported discomfort or difficulties in maintaining the FPP.

\section{Patients With Geotropic LC-BPPV}

At the 24-hour follow-up, 60/78 (76.9\%) of patients undergoing FPP on the correct side had recovered from positional nystagmus and vertigo, compared to $8 / 71$ $(11.3 \%)$ of patients undergoing the sham maneuver $(P<.0001$, Fig. 1). In the geotropic FPP group, positional nystagmus was unchanged (still geotropic) in 16/78 $(20.5 \%)$ patients; in one patient there was a conversion to apogeotropic PN, and in one patient a canal switch to the ipsilateral posterior canal was detected. In the geotropic sham group, positional nystagmus was unchanged (still geotropic) in 54/71 (76.06\%) patients; in five there was a conversion to apogeotropic PN, and in four a canal switch to the ipsilateral PC. Four patients without PN and vertigo at the 24 hours follow-up came back because of a recurrence within a week.

\section{Patients With Apogeotropic LC-BPPV}

At the 24-hour follow-up, 7/38 (18.4\%) of patients undergoing FPP on the correct side had recovered from positional nystagmus and vertigo, compared to 5/34 (14.7\%) of patients undergoing the sham maneuver (Fig. 1, $P=.7583$ ). The apogeotropic variant converted to the geotropic variety in 16 out of 38 patients $(42.1 \%)$ in the treatment group whilst this conversion was observed only in 1 out of 34 patients in the sham group (2.9\%). The cumulative chance of being cured or obtaining a PN transformation was statistically significant between the treatment and sham groups $(P=.0003$, Fig. 1$)$.

Positional nystagmus was unchanged (still apogeotropic) in 12/38 (31.6\%) in the apogeotropic FPP group and in 27/34 (79.4\%) in the sham group. Canal conversion to PC-BPPV was demonstrated in three subjects in the treatment group and one patient in the sham group. In the apogeotropic FPP group, one patient developed a direction fixed PN. Two patients without $\mathrm{PN}$ and vertigo at the 24-hour follow-up returned because of a recurrence within a week.

\section{DISCUSSION}

To the best of our knowledge, this is the first doubleblind randomized trial on the efficacy of the FPP in the treatment of LC-BPPV in both the geotropic and apogeotropic forms. FPP proved highly effective compared to the sham maneuver $(P<.0001)$.

We decided to opt for a short-term (24 hours) study both for ethical reasons and due to the fact that LC-BPPV has a high rate of spontaneous remission within days from onset.

The FPP demonstrated high efficacy in treating the geotropic variant, with a resolution rate of $\mathrm{PN}$ and vertigo at 24 hours in more than $75 \%$ of patients. The rate of PN transformation into the apogeotropic variant is very low ( 1 subject) if the maneuver is performed on the correct side. 
Patients suffering from apogeotropic LC-BPPV have a cumulative chance of being cured or obtaining a PN transformation into geotropic of around 60\%. The high rate of $\mathrm{PN}$ transformation into the geotropic form with the correct maneuver may indicate a canalolithiasis mechanism instead of the generally accepted cupulolithiasis mechanism. Another issue is of course that not all apogeotropic PNs will be converted to a geotropic $\mathrm{PN}$. Intuitively, stuck particles to the cupula may not respond to the FPP, as the velocity generated by gravity alone might not be enough for the particles to move unless a procedure with sufficient force is utilized.

The present study is limited by the short-term follow-up, but as reported above, it is due to ethical reasons and the known benign course of BPPV over days.

The FPP is easily performed and well accepted by patients, especially in severely symptomatic and obese subjects or patients with movement restriction where the GLM may cause harm or be difficult to perform. It could be also effective in reducing the time the patient has to lie on the appropriate side. It probably works because otoconial debris can move into the canal by gravitation. Patient tolerance and compliance to lie on one side for many hours may be another limitation; however, in this series, no patients reported discomfort or difficulties in maintaining the position. Sleeping overnight on one side without turning may be a problem for many.

Unfortunately, at the present time there are insufficient data to establish the relative efficacy of the FPP versus other maneuvers such as the barbecue maneuver. ${ }^{19,20}$ Apart from the GLM, outcomes of most LC-BPPV treatment techniques are based on class IV studies, and the strength of recommendation is not strong. ${ }^{17}$ The GLM seems to be slightly more effective in a similar study. ${ }^{11}$ The main advantage of the GLM over the FPP is the immediate outcome, despite the need for fast acceleration and deceleration while performing the maneuver.

Furthermore, as well as for the GLM, the FPP, with its high rate of $\mathrm{PN}$ transformation from apogeotropic into geotropic PN, suggests an important clue for differential diagnosis between apogeotropic LC-BPPV and central vestibular disorders. The present class 2 study of the efficacy of the FPP changes the level of recommendation of the method for treating LC-BPPV into strong recommendation. ${ }^{17}$

\section{CONCLUSION}

The FPP strategy has to be considered an effective treatment for the geotropic variant of LC-BPPV. ${ }^{14}$ The FPP can also be recommended in cases of the apogeotropic variant, but here it is apparent that the main objective of the procedure would be to convert an apogeotropic variety to a geotropic variety and not a cure.

\section{BIBLIOGRAPHY}

1. von Brevern M, Radtke A, Lezius F, et al. Epidemiology of benign paroxysmal positional vertigo: a population based study. J Neurol Neurosurg Psychiatry 2007;78:710-715.

2. Cho EI, White JA. Positional vertigo: as occurs across all age groups. Otolaryngol Clin North Am 2011;44:347-360.

3. Caruso G, Nuti D. Epidemiological data from 2270 PPV patients. Audiol Med 2005;3:7-11.

4. Nuti D, Vannucchi P, Pagnini P. Benign paroxysmal positional vertigo of the horizontal canal: a form of canalolithiasis with variable clinical features. J Vestib Res 1996;6:173-184.

5. Nuti D, Masini M, Mandalà M. Benign paroxysmal positional vertigo and its variants. In: Furman JM, Lempert T, eds. Handbook of Clinical Neurology. Vol 137. New York, NY: Elsevier; 2016:241-256.

6. von Brevern M, Bertholon P, Brandt T, et al. Benign paroxysmal positional vertigo: diagnostic criteria. $J$ Vestib Res 2015;25:105-117.

7. Mandalà M, Salerni L, Nuti D. Benign positional paroxysmal vertigo treatment: a practical update. Curr Treat Options Neurol 2019;21:66.

8. Lempert T. Horizontal benign positional vertigo. Neurology 1994;44: 2213-2214.

9. Kim JS, Oh SY, Lee SH, et al. Randomized clinical trial for apogeotropic horizontal canal benign paroxysmal positional vertigo. Neurology 2012;78: 159-166.

10. Kim JS, Oh SY, Lee SH, et al. Randomized clinical trial for geotropic horizontal canal benign paroxysmal positional vertigo. Neurology 2012;79. 700-707.

11. Mandalà M, Pepponi E, Santoro GP, et al. Double-blind randomized trial on the efficacy of the Gufoni maneuver for treatment of lateral canal BPPV. Laryngoscope 2013;123:1782-1786.

12. Vannucchi P, Giannoni B, Pagnini P. Treatment of horizontal semicircular canal benign paroxysmal positional vertigo. $J$ Vestib Res 1997;7:1-6.

13. Nuti D, Agus G, Barbieri MT, Passali D. The management of horizontalcanal paroxysmal positional vertigo. Acta Otolaryngol 1998;118:455-460.

14. Casani AP, Vannucci G, Fattori B, Berrettini S. The treatment of horizontal canal positional vertigo: our experience in 66 cases. Laryngoscope 2002 ; 112:172-178.

15. Vannucchi P, Asprella Libonati G, Gufoni M. The physical treatment of lateral semicircular canal canalolithiasis. Audiol Med 2005;3:52-56.

16. Fife TD, Iverson DJ, Lempert T, et al. Practice parameter: therapies for benign paroxysmal positional vertigo (an evidence-based review): report of the Quality Standards Subcommittee of the American Academy of Neurology. Neurology 2008;70:2067-2074.

17. Bhattacharyya N, Baugh RF, Orvidas L, et al. American Academy of Otolaryngology-Head and Neck Surgery Foundation. Clinical practice guideline: benign paroxysmal positional vertigo. Otolaryngol Head Neck Surg 2008;139:S47-S81.

18. Strupp M, Mandalà M, López-Escámez JA. Peripheral vestibular disorders: an update. Curr Opin Neurol 2019;32:165-173.

19. Ichijo $H$. A new treatment (the affected-ear-up $90^{\circ}$ maneuver) for benign paroxysmal positional vertigo of the lateral semicircular canal. Acta Otolaryngol 2019;139:588-592.

20. Faria Ramos B, Calc R, Martins Brock C, Mangabeira Albernaz PL Zuma E, Maia F. Apogeotropic variant of horizontal Semicircular Canal benign paroxysmal positional vertigo: where are the particles? Audiol Res 2019;29:228. 